\title{
Kleines Auslandsstudium zu Hause: Chancen und Grenzen des digitalen Lernens am Fall des japanisch-deutschen Online-Seminars „Global Medial“
}

\begin{abstract}
Zusammenfassung
Im Folgenden wird ein Modellprojekt eines online-basierten Hochschulseminars aus japanischer Perspektive vorgestellt. Im Seminar geht es um den Kompetenzerwerb deutscher und japanischer Studierender im Umgang mit Globalisierung und Digitalisierung. Die auf Deutsch durchgeführte Lehrveranstaltung fungierte für die japanischen Studierenden als ein kleines Auslandsstudium zu Hause.
\end{abstract}

Schlüsselworte: Globales Lernen, Medienkompetenz, Japan, Digitales Lernen

\begin{abstract}
The following article describes a project of an online-based seminar from a Japanese viewpoint. It is very important for students in Germany and Japan to develop their competence in the seminar especially focusing on globalization and digitalization. This seminar provided a small experience of studying at a foreign university to Japanese students without going abroad.
\end{abstract}

Keywords: Global Learning, Media Competence, Japan, Digital Learning

\section{Einleitung}

Dieser Beitrag betrachtet, welche Lernerfahrungen japanische Studierende in dem deutsch-japanischen Online-Hochschulseminar „Global Medial“ gemacht haben. Zwar wurde bereits ein Bericht über dieses Seminar veröffentlicht (Stratmann, Lang-Wojtasik \& Erichsen-Morgenstern, 2018); an dieser Stelle wird die Lehrveranstaltung nochmal aus japanischer Perspektive reflektiert. Die Veranstaltung „Global Medial“ wurde als ein Modellprojekt mit Teilnahme von Studierenden aus Deutschland (Pädagogische Hochschule Weingarten) und aus Japan (Hiroshima City University) im Wintersemester 2017/18 durchgeführt. Im Seminar wurden insbesondere zwei gesellschaftliche Veränderungen und Herausforderungen behandelt: Globalisierung und Digitalisierung.

In Bezug auf die mit Globalisierung und Digitalisierung einhergehenden Prozesse werden international unterschiedliche, neue gesellschaftliche Konzepte vorgeschlagen, wie z.B.: „Industrie 4.0" in Deutschland, "Industrial Internet" in den USA, „e-Estonia“ in Estland oder „Smart Nation“ in Singapur. In diesem Zusammenhang schlägt auch die japanische Regierung eine gesamtgesellschaftliche Initiative der „Society 5.0“ (Gesellschaft 5.0) vor. Sie zielt auf eine superdigitalisierte, aber auch eine menschliche Gesellschaft (engl. Super Smart Society) ab, in der sich Wirtschaftswachstum und gesellschaftliche Problemlösung durch hochkombinierte Systeme zwischen Cyberspace (virtueller Raum) und physikalischem bzw. wirklichem Raum miteinander vereinbaren lassen (Government of Japan, 2016). Dabei wird einerseits erwartet, dass die durch Sensoren und Geräte gesammelten Daten als riesige Datenmengen im virtuellen Raum gespeichert werden sollen, damit die Daten mithilfe künstlicher Intelligenz analysiert werden können. Andererseits sollen Wirtschaftswachstum und gesellschaftliche Problemlösung im physikalischen Raum mit Hilfe von Maschinen und Robotern inkl. künstlicher Intelligenz realisiert werden.

Vor diesem Hintergrund hat sich die Provinz Hiroshima entschieden, im Jahr 2019 eine neue interkulturelle Oberschule auf einer entvölkerten Insel zu gründen. Es wird geplant, global kompetente Schüler/-innen mit einem international anerkannten Schulabschluss, dem „International Baccalaureate“, durch den Austausch mit Schüler/-innen aus der ganzen Welt weiterzubilden. Dabei sollen alle Schüler/-innen während der Schulzeit ein tragbares Endgerät nutzen, damit ihre Daten kontinuierlich gespeichert und analysiert werden können (Chugoku-Shinbun, 2018, S. 17). Hier wird eine radikale Kombination von Globalisierung und Digitalisierung in der Schule ersichtlich. Auch bei der folgend vorgestellten online-basierten Lehrveranstaltung zwischen einer deutschen und einer japanischen Hochschule wird sowohl Globalisierung als auch Digitalisierung thematisiert, allerdings in zeitlich und räumlich begrenzter Weise. Dazu soll zunächst das Seminar „Global Medial“ kurz zusammenfassend dargestellt werden. Anschließend werden Globales Lernen (Fokus: Globalisierung) und Medienkompetenz (Fokus: Digitalisierung) aus japanischer Sicht reflektiert und damit eine nicht-eurozentrierte Beobachtungshaltung eingenommen. Zum Schluss werden die Chancen und Grenzen des digitalen Lernens diskutiert. 


\section{Das interkontinentale digitalisierte Seminar „Global Medial” \\ Kurze Beschreibung des Seminars}

Das Seminar „Global Medial“ zielte auf den Kompetenzerwerb im Bereich des Globalen Lernens und der Medienpädagogik in einer verbundenen Form ab. Dabei wurde von den Studierenden erwartet, mit den Herausforderungen einer Weltgesellschaft kommunikativ umzugehen. Diese Veranstaltung gehörte zu einem e-Learning Angebot (elektronisch unterstütztes Lernen). Als offizielle Sprache des Seminars wurde Deutsch ausgewählt, wobei auch Englisch im Bedarfsfall gesprochen werden konnte. In Deutschland hat sich das Lehrangebot ausschließlich an Studierende erziehungswissenschaftlicher Studiengänge am Ende der Bachelor-Phase und am Beginn der Master-Phase gerichtet, während es in Japan den Bachelor-Studierenden mit guten Deutschkenntnissen aber mit unterschiedlichsten Fachinteressen in der Fakultät für Internationale Studien angeboten wurde. Außerdem wurde das Seminar in Deutschland als ein reines Online-Lernen veranstaltet (Stratmann, Lang-Wojtasik \& Erichsen-Morgenstern, 2018, S. 30), während es in Japan mit allen zusammen in einem Raum unserer Hochschule durchgeführt wurde, damit auf sprachliche oder technische Störungen sofort reagiert werden konnte.

Das Seminar bestand sowohl aus einigen synchronen Onlinetreffen als auch aus verschiedenen asynchronen Selbstlernphasen, die in einen Takt verpackt wurden. $\mathrm{Zu}$ einem Takt gehörten folgende vier Phasen: 1) Onlinetreffen zum Bereitstellen neuer Lehrinhalte, 2) Asynchrone Lernphase in Gruppen, 3) Synchrone Treffen für Fragen und 4) Onlinetreffen zur Konferenz mit Präsentationen. Die Onlinetreffen sowie die synchronen Treffen für Fragen fanden immer am Dienstag (8:20 - 9:50 Uhr in Deutschland / 16:20 - 17:50 Uhr in Japan) statt. Auch wenn sich die technische Lernumgebung weiterentwickeln wird, ist es leider immer noch unmöglich, den Zeitunterschied zwischen den Ländern zu überwinden.

Als Einführung in das Seminar sollten die Studierenden zuerst einen Video-Steckbrief zu ihrer Person anfertigen. Hierdurch wurde das Gegenüber als Person mit bestimmten Zielen und Interessen wahrgenommen, wodurch Austauschprozesse initiiert und das Zusammenfinden der internationalen Kleingruppen erleichtert werden konnte (ebd., S. 31). Dadurch wurden drei international gemischte Studierendengruppen gebildet, um den netzbasierten Austausch zu fördern: die insgesamt drei Gruppen setzten sich also im Verhältnis aus deutschen und japanischen Studierenden folgendermaßen zusammen: 1. Gruppe: vier/zwei, 2. Gruppe: zwei/drei, 3. Gruppe: vier/zwei. Beim synchronen Onlinetreffen sollten sich alle in die Software „Adobe Connect“ einloggen, während alle Lernmaterialen für die Vorbereitungen in der „Moopaed“ (einer Lernplattform der Pädagogischen Hochschule Weingarten) verfügbar waren. In der asynchronen Lernphase haben die Studierenden auch online meistens durch WhatsApp oder LINE (eine japanische WhatsApp-Variante) und ggf. per Skype zwischen den Kontinenten kommuniziert.

In der Veranstaltung wurde eine Sequenzierung des Lernprozesses durch eine zeitliche Taktung vorgenommen und es gab insgesamt sechs Takte/Lerneinheiten vor der Durchführung einer Abschlusssitzung. In jeder Lerneinheit wurde von allen Studierenden erwartet, mindestens einen Studientext des jeweiligen Themas gelesen zu haben. Die Bedeutung des Themas und ihre Lernaufgaben in dem Takt konnten durch ein Lehrvideo der jeweiligen Lehrperson im „Moopaed“ vorbereitet werden. Die Lernaufgaben zielten auf eine kooperative Bearbeitung des jeweiligen Themas innerhalb der internationalen Gruppen ab und manifestieren sich i. d. R. in einem gemeinsamen Produkt, das in einer Online-Konferenz präsentiert und im Online-Plenum diskutiert wurde (ebd., S. 32).

Glücklicherweise hatten wir kein Drop-Out und auch keine schwere technische Störung. Die Qualität der Präsentationen entsprach weitgehend den vorab vermuteten Erwartungen (vgl. ebd.). In den Diskussionen dominierten die deutschen Studierenden, wie bei einem Heimspiel, was in den Rückmeldungen besonders auf sprachliche Herausforderungen zurückgeführt wurde. Für die japanischen Teilnehmenden war das Seminar hingegen stärker mit dem Gefühl von einem Auswärtsspiel verbunden.

\section{Globalisierung und Medienkompetenz: \\ Was haben Japanische Studierende gelernt?}

Im Seminar wurde insbesondere behandelt, wie Studierende mit Globalisierung und digitalen Medien umgehen und welcher Kompetenzerwerb dabei bedeutsam ist (ebd., S. 29). Für diese Fragestellung interessierten sich auch die japanischen Studierenden, weil das Problem eigentlich eine gemeinsame Herausforderung weltweit darstellt.

In der Beschäftigung mit Globalem Lernen ging es darum (ebd., S. 31)

- die Bedeutung der Globalisierung und Entwicklung hin zur Weltgesellschaft für Kommunikationsprozesse in Ansätzen zu verstehen und auf Lernarrangements beziehen zu können;

- $\quad$ sich mit theoretischen Grundlagen des interdisziplinären Diskursfeldes vertraut zu machen und diese auf eigene Fragestellungen zu beziehen;

- aktuelle Konzeptionen und Kompetenzdebatten kennenzulernen und auf ihre Tragfähigkeit in eigenen Reflexionsund Handlungsfeldern zu prüfen;

- Handlungsfelder über den eigenen Handlungskontext hinaus kennenzulernen und an eigene Aktivitäten anzuschließen;

- sich mit der bildungspolitischen Relevanz des Themenfeldes zu beschäftigen.

$\mathrm{Zu}$ Beginn wurde ersichtlich, dass die japanischen Studierenden eine andere Vorstellung von Globalisierung, Internationalisierung und Globalem Lernen haben als die deutschen Studierenden. Es wurde erkennbar, dass die japanischen Studierenden Globales Lernen verstärkt als wirtschaftspädagogische Lernprozesse in Bezug auf Öffnung der Grenzen und internationale Wirtschaftskooperation und damit verbundene ökonomische Wettbewerbsvorteile verstehen. Das Seminar aber hat die japanischen Studierenden zu einer anderen Perspektive und einer neuen Denkweise in Bezug auf Globales Lernen angeregt.

Am Anfang konnten unsere Studierenden eigentlich nicht verstehen, warum sie überhaupt am Seminar zum Globalen Lernen teilnehmen sollten. In unserer Fakultät für Internationale Studien können alle Studiengänge selbstverständlich jeweiliges Grundwissen fast aller Human- und Sozialwissen- 
schaften interdisziplinär anbieten, d.h.: alle Studierenden werden bereits im Studium automatisch mit dem Themenfeld des Globalen Lernens (in ihrem Verständnis) konfrontiert. Wozu sollten japanische Studierende der Fakultät für Internationale Studien also eine explizite Lehrveranstaltung zum Globalen Lernen besuchen, wo doch fast alle Lerninhalte des Studiums bereits auf die Themenbereiche des Globalen Lernens bezogen werden können? Im Laufe des Seminars wurde von den Studierenden immer implizit versucht, auf diese Frage eine Antwort zu finden, die aber interessanterweise nicht gefunden wurde. Aber die japanischen Studierenden haben durch diese Veranstaltung ihr bisheriges Verständnis zum Globalen Lernen grundlegend erweitert und gelernt, dass die normativen Horizonte von Nachhaltigkeit und Gerechtigkeit im Hinblick auf Globalisierung beim Globalen Lernen in Deutschland eine wichtige Voraussetzung sind.

In Bezug auf die Medienkompetenz wurde auf die Entwicklung von Sach-, Sozial- und Selbstkompetenzen der Studierenden hingewiesen (vgl. ebd., S. 31). Sie sollten:

- die Bedeutung von Medienkompetenz in einer von (digitalen) Medien durchdrungenen Gesellschaft erläutern und sich sicher in ihrer medialen Umwelt bewegen und diese so gestalten, dass ihre Bedürfnisse befriedigt werden (Sachbezug);

- den Einfluss von Medien auf den Kommunikationsprozess erläutern und medienvermittelte Kommunikationsprozesse den Anforderungen entsprechend gestalten (Sozialbezug);

- den Einfluss von Medien auf den Sozialisationsprozess erläutern und ihre eigene mediale Umwelt daraufhin reflektieren und (digitale) Medien für ihre eigene Persönlichkeitsentwicklung auswählen und verwenden (Selbstbezug).

Hinsichtlich der Medienkompetenz geht es für japanische Studierende um eine praktische Gebrauchsfähigkeit eines Gerätes (hauptsächlich PC) oder einen Umgang mit Softwares (i.d.R. Word, Excel, Power Point und eine interne Lernplattform der Hochschule), weil immer weniger Studierende heute einen eigenen PC haben und sie ein verpflichtendes Training der PC-Anwendung mit einem grundlegenden Wissen für ihr Studium brauchen.

Im Seminar haben die japanischen Studierenden jedoch im Bereich der Medienkompetenz eine andere noch wichtigere Dimension kennengelernt, nämlich dass der Begriff der Kompetenz sich heute als ein Kernbegriff der Erziehungswissenschaft weltweit verbreitet und eine zunehmend wichtigere Rolle spielt. Dadurch erweitertete sich das Kompetenzverständnis der japanischen Studierenden und sie konnten im Seminar den aktuellen Kompetenzdiskurs im internationalen Kontext (z.B. DeSeCo der OECD) sowie zum Globalen Lernen kennenlernen und mit den deutschen Studierenden diskutieren und reflektieren.

\section{Zugang zur Wissenschaft mit außerenglischer Sprachkompetenz}

Eigentlich sollte das Seminar ausschließlich auf Englisch durchgeführt werden, da diese Veranstaltung mit der Annahme geplant wurde, dass deutsche, japanische und indische Studierende teilnehmen und zusammenarbeiten würden. Aber leider sind die indischen Teilnehmenden kurz vor Beginn der Veranstaltung aus organisatorischen Gründen abgesprungen. Das führte zu einer Veränderung in der sprachlichen Fokussierung von Englisch auf Deutsch. Daraus ergab sich ein kommunikativer Nachteil der japanischen Studierenden, den sie aber auch als Vorteil nutzen konnten. Denn sie hatten die Gelegenheit, ihre Deutschkenntnisse zu verbessern und so funktionierte das Seminar als ein kleines Auslandsstudium im eigenen Land.

\section{Entmonopolisierung der englischsprachigen Lernumgebung durch eine zweite Fremdsprache}

Zufälligerweise konnten vier von sieben japanischen Studierenden bereits genügend Deutsch sprechen, weil sie gerade nach ihrem Austauschstudium für ein halbes oder ein ganzes Jahr aus Deutschland zurückgekommen waren. Unsere Fakultät für Internationale Studien verpflichtet alle Studierenden das Lernen zweier Fremdsprachen (Deutsch, Französisch, Spanisch, Italienisch, Russisch, Arabisch, Chinesisch, oder Koreanisch), während manche japanische Hochschulen ausschließlich eine Fremdsprache, normalerweise Englisch, unterrichten. Damit können unsere Studierenden eine Chance zum Auslandsstudium nicht nur in englischsprachigen Ländern, sondern auch in acht anderen Sprachräumen erhalten. Das ist ein wichtiger Baustein, um die Dominanz von Englisch als einziger Fremdsprache aufzuweichen und Studierende auch für andere Sprachen zu begeistern. Und genau das ist auch durch das Seminar „Global Medial“, das in deutscher Sprache durchgeführt wurde, gelungen. Bei uns heißt das Seminar eigentlich „Gesellschaft in Deutschland“ statt „Global Medial“. Mit einer Reflexion des Seminars konnte der sprachliche Nachteil japanischer Studierende dann auch als ein Beitrag zur Möglichkeit der Entmonopolisierung englischsprachiger Lernumgebungen interpretiert werden. Japanische Studierende könnten also einen Zugang zu einer anderen Welt bzw. Kultur dadurch erlangen, dass sie eine zweite Fremdsprache intensiver lernen oder vertiefen können, wie es im Seminar der Fall war.

\section{Vom Lernen zum Studieren mit deutschen wissenschaftlichen Verhältnissen}

Außerdem konnten die japanischen Studierenden jeweils ihr eigenes Studium durch das Seminar „Global Medial“ nochmals reflektieren bzw. relativieren. Wie oben bereits erwähnt, hatten unsere Studierenden nicht wirklich verstanden, wozu sie ein Seminar zum Globalen Lernen besuchen sollten. Aber das Seminar eröffnete ihnen dann doch eine bedeutsame Reflexionsmöglichkeit, verknüpft mit der Frage, welche Inhalte und Perspektiven, die bisher nicht betrachtet wurden, bei einem international ausgerichteten Studium relevant sein können.

Das in Japan als eine Lehrveranstaltung mit dem Titel „Gesellschaft in Deutschland“ durchgeführte Seminar „Global Medial“ führte nämlich bei den japanischen Studierenden zu neuen Erlebnissen im Vergleich mit deutschen inhaltlichen sowie methodischen Lernritualen, d.h.: 1) das deutsche Globale Lernen und 2) die wissenschaftlichen Verhältnisse einer deutschen Hochschule.

In Japan wird Globales Lernen oft als eine Erziehung zur globalen Kompetenz im Hinblick auf die Entwicklung von Humankapital interpretiert (Urabe, 2017, S. 188). Dabei wird implizit angenommen, dass man eine gute Arbeitsleistung im 
internationalen wirtschaftlichen Wettbewerb erlangen soll. In diesem Zusammenhang geht es den Schüler/inne/n sowie Studierenden in Japan dann auch verstärkt um das Training ihrer englischen Sprachkompetenz. Allerdings wird zugleich von den japanischen Studierenden auch erwartet, dass sie möglichst viele der verpflichtenden Seminarscheine im Studium möglichst schnell erhalten, um ihre Arbeitssuche am Ende des Studiums zu konzentrieren. Im japanischen Arbeitsmarkt gibt es eine bestimmte Bewerbungssaison, innerhalb der die Studierenden eine vorläufige Stellenzusage als Vollzeitbeschäftigte eines Unternehmens bekommen sollen. Daher erfolgt das Studium in Japan in der Regel relativ zügig und meistens steht die schnelle und reine Informationsübermittlung von Fachwissen im Vordergrund. Oftmals bleiben keine Freiräume für die Reflexion der Lerninhalte, theoretische Überlegungen oder lebhafte Diskussionen.

Vor diesem Hintergrund haben die japanischen (und vielleicht auch deutschen) Studierenden im Seminar „Global Medial“ die Chance genutzt, miteinander zu diskutieren, zu reflektieren und dabei zu erkennen, dass Globalisierung nicht auf Internationalisierung reduziert werden kann, sondern auch mit Kernbegriffen wie Nachhaltigkeit und Gerechtigkeit normativ verstanden werden kann und soll. Damit haben die japanischen Teilnehmenden im interkontinentalen Vergleich gelernt, dass Globales Lernen theoretisch interpretiert sowie vielschichtiger und breiter verstanden werden kann als sie es bisher taten.

In Bezug auf die japanische Diskussion zu den globalen humanen Ressourcen versuchte eine Studierendengruppe Beziehungen zwischen Menschenbildern und Kompetenzen mit einem theoretischen Rahmen zu analysieren. Diese operationalisierte wissenschaftliche Denkweise war eigentlich ganz neu für die japanischen Studierenden. Denn durch die interkontinentale Diskussion wurde von Studierenden präsentiert, dass Globales Lernen in Japan kaum theoretisch fundiert wurde. Durch das Seminar konnten die japanischen Studierenden eine Veränderungsmöglichkeit in Bezug auf Lernprozesse im Studium erkennen. Sie konnten nämlich wahrnehmen, dass Lernen als eine reine Sammlung neuer Information (wie sie es bisher vornehmlich kannten) im Studium durch theoretische Reflexionsprozesse erweitert werden kann. Anders formuliert, die japanischen Studierenden haben also deutschlandspezifisch akademische bzw. wissenschaftliche Verhältnisse mit theoretischer Operationalisierung durch das Seminar "Global Medial“ kennengelernt.

\section{Schlussfolgerung: Chancen und Grenzen des digitalen Lernens}

Durch die gemeinsame online-basierte Lehrveranstaltung haben alle (deutsche und japanische) Studierende eine erziehungswissenschaftliche Grundlage zum Globalen Lernen und zur Medienkompetenz kennengelernt. Aus japanischer Sicht liegen die Chancen der digitalen Lernumgebung und die positiven Lerneffekte in drei Bereichen.

1. Zunächst war das digitale Lernen eine gelungene Übungsgelegenheit sowie Verbesserungsmöglichkeit in Bezug auf das Erlernen der Fremdsprache Deutsch. Damit leistete das Seminar „Global Medial“ einen wichtigen Beitrag dazu, den japanischen Studierenden die Vertiefung einer Fremdsprache jenseits des oftmals dominierenden Englischen zu ermöglichen.
2. Mit genügender Sprachkompetenz führte das digitale Lernen als nächstes zu einer zeitlich unbegrenzten bzw. asynchronen Vorbereitungschance auf einer online-gestützten Lernplattform, damit alle Studierenden z.B. über Kernbegriffe zu globaler Kontextualisierung im synchronen Lernen intensiver diskutieren konnten. Im Seminar „Global Medial“ sollten einige Grundbegriffe, z.B. Globalisierung und Kompetenz beispielsweise vorher online bearbeitet werden, bevor Globales Lernen sowie Medienkompetenz im internationalen Zusammenhang diskutiert wurden.

3. Außerdem ermöglichte das digitale Lernen unterschiedliche Kombinationschancen mit den internationalen/intranationalen und heterogenen/homogenen Lerngruppen, ohne an einem Ort anwesend zu sein. Damit konnten wichtige interkulturelle Reflexions- und Relativierungsprozesse eröffnet werden. Denn in der Lehrveranstaltung wurden Globales Lernen und Medienkompetenz sehr unterschiedlich verstanden. Dadurch konnten die verschiedenen Beobachtungsperspektiven von deutschen und japanischen Studierenden interaktiv und dynamisch ausgetauscht und interpretiert werden.

Zuletzt soll auch auf zwei Grenzen des digitalen Lernens hingewiesen werden. Im digitalen Lernen findet man hauptsächlich zeitliche und technische Grenzen. Die zeitliche Grenze ist geographisch bedingt: Obwohl sich die Informationstechnologie immer weiter entwickeln konnte, müssen wir alle trotzdem den Zeitunterschied zwischen den Ländern berücksichtigen. Während man im Bett schläft, sollte ein digitales Lernen insbesondere ein synchrones Onlinetreffen nicht stattfinden. Die technische Grenze hängt von der Lernumgebung bzw. Infrastruktur ab. Im Seminar „Global Medial“ wäre es unmöglich gewesen, wenn keiner z.B. mit der Software „Adobe Connect“ hätte operieren könnte. Und es wurde auch erwartet und angenommen, dass alle Seminarteilnehmenden jeweils ein Endgerät wie PC, Tablet oder Smartphone mit einem Internetanschluss haben. Hier offenbaren sich Nachteile für diejenigen, die über solche technische Rahmenbedingungen gar nicht oder nur eingeschränkt verfügen.

\section{Literatur}

Chugoku-Shinbun (2018). Tragbares Endgerät für alle Schülerinnen und Schüler In Tageszeitung Chugoku-Shinbun am Montag, 30. April 2018. Hiroshima, 17.

Government of Japan (2016). The 5th Science and Technology Basic Plan. Tokyo: Cabinet Office, Goverment of Japan.

Stratmann, J., Lang-Wojtasik, G. \& Erichsen-Morgenstern, R. M. (2018). Global Medial - Kompetenzerwerb von Studierenden in einer interkulturellen online bereitgestellten Lernumgebung. Zeitschrift für internationale Bildungsforschung und Entwicklungspädagogik, 41(1), 29-33.

Urabe, M. (2017). Globales Lernen in Japan. In G. Lang-Wojtasik \& U. Klemm (Hrsg.), Handlexikon Globales Lernen (2. Aufl., S. 187-191). Münster \& Ulm: Klemm + Oelschläger.

\section{Dr. Masashi Urabe}

ist Associate Professor für Erziehungswissenschaft an der Hiroshima City University, Japan. Arbeits- und Forschungsschwerpunkte: Vergleichende und Internationale Erziehungswissenschaft und Bildung für nachhaltige Entwicklung. 